\title{
QUASI-COMPLEMENTED ALGEBRAS
}

BY

\section{T. HUSAIN( $\left.{ }^{1}\right)$ AND PAK-KEN WONG ${ }^{2}$ )}

\begin{abstract}
In this paper we introduce a class of algebras which we call quasi-complemented algebras. A structure and representation theory is developed. We also study the uniformly continuous quasi-complementors on $B^{*}$-al gebras.
\end{abstract}

1. Introduction. Complemented Banach algebras were introduced in [11] and have been studied by various authors. The present work is an attempt to generalize these algebras.

The concept of quasi-complemented algebra is introduced in $\$ 2$. Let $A$ be a semisimple quasi-complemented algebra in which every maximal modular right ideal is closed. We show that the socle of $A$ is dense in $A$. This enables us to establish a structure theorem for $A$ if $A$ has the property $x \in \operatorname{cl}(x A)$ for all $x \in A$. We also give a representation theorem for a primitive Banach algebra in which every maximal closed right ideal is modular and $x \in \operatorname{cl}(x A)$ for all $x \in A$. In $\$ 5$, we study quasi-complementors induced by given quasi-complementors.

We introduce the concept of continuous quasi-complementors in $\$ 6$. Then we show that if $A$ is a $B^{*}$-algebra which has no minimal left ideals of dimension less than three, then every uniformly continuous quasi-complementor on $A$ is a complementor.

As we observed above, many fundamental properties of a complemented algebra hold for a quasi-complemented algebra. However a quasi-complemented algebra, in general, is not complemented as shown by the examples in $\$ 2$.

2. Notation and preliminaries. For any subset $S$ in an algebra $A$, let $l(S)$ and $r(S)$ denote the left and right annihilators of $S$ in $A$, respectively. Let $A$ be a topological algebra. Then $A$ is called an annihilator algebra if, for every closed left ideal $J$ and for every closed right ideal $R$, we have $r(J)=(0)$ if and only if $J=A$ and $l(R)=(0)$ if and only if $R=A$. If $l(r(J))=J$ and $r(l(R))=R$, then $A$ is called a dual algebra.

Received by the editors December 16, 1971.

AMS (MOS) subject classifications (1969). Primary 4650; Secondary 4655.

Key words and phrases. Quasi-complemented algebras, annihilator and dual algebra, complemented algebra, continuous and uniformly continuous quasi-complementors.

(1) This work was supported by a N.R.C. grant.

(2) The second author was supported by a postdoctoral fellowship at McMaster University. 
Let $A$ be a topological algebra and let $L_{r}$ be the set of all closed right ideals in $A$. Then $A$ is called a right quasi-complemented algebra if there exists a mapping $q: R \rightarrow R^{q}$ of $L_{r}$ into itself having the following properties:

(2.1) $R \cap R^{q}=(0)\left(R \in L_{r}\right)$;

(2.2) $\left(R^{q}\right)^{q}=R\left(R \in L_{r}\right)$;

(2.3) if $R_{1} \supset R_{2}$, then $R_{2}^{q} \supset R_{1}^{q}\left(R_{1}, R_{2} \in L_{r}\right)$.

We call the mapping $q$ a right quasi-complementor on $A$ and $R^{q}$ the right quasi-complement of $R$ in $A$. It is clear that the concept of quasi-complementation extends that of orthogonal complementation when $A$ is a Hilbert algebra.

A right quasi-complemented algebra $A$ is called a right complemented algebra if it satisfies:

(2.4) $R+R^{q}=A\left(R \in L_{r}\right)$

In this case, the mapping $q$ is called a right complementor on $A$ (see [11, p. 615, Definition 1]). A right quasi-complemented algebra may not be right complemented as shown by the following examples:

Example 2.1. Let $B$ and $p$ be given in [1, p. 396, Example 1]. Then $p$ is a right quasi-complementor on $B$. But $p$ is not a right complementor. However $B$ is a right complemented a lgebra under the right complementor $R \rightarrow l(R)^{*}$ (see [3, p. 463, Theorem 3.6]).

Example 2.2. Let $G$ be the compact group of real numbers mod 1 and $A=$ $L_{p}(G)$, where $1<p<\infty$ and $p \neq 2$. It is well known that $A$ is a commutative dual $A^{*}$-algebra which is not an ideal in the completion of its auxiliary norm (see [9, p. 35]). By Theorem 6.5, the mapping $q: R \rightarrow l(R)$ is the only right quasi-complementor on $A$. It follows from [4, p. 233, Theorem 3.8] and [9, p. 35, Theorem 23] that $p$ is not a right complementor on $A$. Since $A$ has a unique right quasi-complementor, $A$ is not a right complemented algebra.

Analogously we define left quasi-complemented algebras. In this paper, we limit our attention to right quasi-complemented algebras with the remark that similar properties hold for left quasi-complemented algebras. From now on a quasi-complemented (resp. complemented) algebra will always mean a right quasicomplemented (resp. right complemented) algebra.

Let $X$ be a topological space and $S$ a subset in $X$. Then $\mathrm{cl}(S)$ will denote the closure of $S$ in $X$.

In this paper, all algebras and linear spaces under consideration are over the complex field $C$. Definitions not explicitly given are taken from Rickart's book [10].

We shall need the following result.

Lemma 2.1. Let $A$ be a semisimple dual algebra in which every maximal modular right ideal is closed. Then for each nonzero closed right ideal $R$ of $A$, 
we have $R=\operatorname{cl}\left(\Sigma_{a} e_{\alpha} A\right)$, where $\left\{e_{\alpha}\right\}$ is the family of all minimal idempotents of $A$ contained in $R$.

Proof. By [5, p. 569, Theorem 4.2], $\left\{e_{a}\right\}$ is not an empty set. Let $J=$ $\operatorname{cl}\left(\Sigma_{a} e_{a} A\right)$. By a similar argument in the proof of [5, p. 570, Theorem 5.1], we have $l(J) R=(0)$ and so $R \subset r(l(J))=J$. Therefore $R=J$. This completes the proof.

\section{A structure theorem.}

Lemma 3.1. Let $A$ be a quasi-complemented algebra with a quasi-complementor q. Then

(i) For any family of closed right ideals $\left\{R_{\lambda}\right\}$ in $A$, we bave $\operatorname{cl}\left(\Sigma_{\lambda} R_{\lambda}\right)=$ $\left(\bigcap_{\lambda} R^{q}\right)^{q}$.

(ii) For every closed right ideal $R$ of $A, R+R^{q}$ is dense in $A$.

Proof. (i) follows from the proof of [3, p. 461, Lemma 2.1].

(ii) Since $A^{q}=A^{q} \cap A=(0)$, we have $(0)^{q}=A$. Therefore it follows from (i) that

$$
\operatorname{cl}\left(R+R^{q}\right)=\left(R^{q} \cap R\right)^{q}=(0)^{q}=A .
$$

Therefore $R+R^{q}$ is dense in $A$.

Corollary 3.2. A finite dimensional quasi-complemented normed algebra is a complemented algebra.

Proof. This follows easily from Lemma 3.1(ii).

Lemma 3.3. Let $A$ be a semisimple quasi-complemented algebra in which every maximal modular right ideal is closed. Then the socle of $A$ is dense in $A$.

Proof. Let $\left\{R_{\lambda}: \lambda \in \Lambda\right\}$ be the family of all maximal modular right ideals of $A$. By the semisimplicity of $A, \bigcap_{\lambda} R_{\lambda}=(0)$ and therefore by Lemma 3.1, $A=$ $\operatorname{cl}\left(\Sigma_{\lambda} R_{\lambda}^{q}\right)$. Clearly $R_{\lambda}^{q} \neq(0)$; for otherwise $R_{\lambda}=\left(R_{\lambda}^{q}\right)^{q}=(0)^{q}=A$, a contradiction. Since $R_{\lambda}+R_{\lambda}^{q}$ is a right ideal which contains $R_{\lambda}$ properly, it follows that $R_{\lambda}+R_{\lambda}^{q}=A$. Therefore by Lemma 3.1 in [7], $R_{\lambda}^{q}$ is a minimal right ideal. Hence $R_{\lambda}^{q}$ is contained in the socle $S$ of $A$ and therefore $S$ is dense in $A$. This completes the proof.

Lemma 3.4. Let $A$ be a semisimple quasi-complemented algebra sucb that $x \in \operatorname{cl}(x A)$ for all $x \in A$. Then each closed two-sided ideal $J$ in $A$ is a quasicomplemented algebra.

Proof. Let $R$ be a closed right ideal in $J$. Since $l(J)=r(J)$ (see [14, p. 37]) and $J^{q} J \subset J \cap J^{q}=(0)$, it follows that $J^{q} \subset l(J)=r(J) \neq(0)$. Therefore 
by the proof of $[10$, p. 99 , Lemma (2.8.11)], $R$ is a closed right ideal in $A$. Let $q$ be a given quasi-complementor on $A$ and let $R^{q J}=R^{q} \cap J$. We show that $q_{J}$ is a quasi-complementor on $J$. By Lemma 3.1, we have

$$
\left(R^{q J}\right)^{q J}=\left(R^{q} \cap J\right)^{q} \cap J=\mathrm{cl}\left(R+J^{q}\right) \cap J .
$$

Let $x \in\left(R^{q J}\right)^{q J}$ and write $x=\lim _{\alpha}\left(a_{a}+b_{\alpha}\right)$ with $a_{\alpha} \in R$ and $b_{\alpha} \in J^{q}$. Since $x \in J$, it follows from Lemma 3.1 that

$$
x A=x \operatorname{cl}\left(J+J^{q}\right) \subset \operatorname{cl}\left(x\left(J+J^{q}\right)\right)=\operatorname{cl}(x J) .
$$

Since $x \in \operatorname{cl}(x A)$, we have $x \in \operatorname{cl}(x J)$. Therefore we can write $x=\lim _{\beta} x y_{\beta}$ with $y_{\beta} \in J$. Since

$$
x y_{\beta}=\lim _{a}\left(a_{a} y_{\beta}+b_{\alpha} y_{\beta}\right)=\lim _{a} a_{\alpha} y_{\beta},
$$

it follows that $x y_{\beta} \in R$ and consequently $x \in R$. Therefore $\left(R^{q J}\right)^{q J} \subset R$. Since $R^{q} \cap J \subset R^{q}$, we have $R \subset\left(R^{q J}\right)^{q J}$ and hence $\left(R^{q J}\right)^{q J}=R$. It is easy to see that the mapping $q_{J}$ satisfies the conditions (2.1) and (2.3). Therefore it is a quasi-complementor on $J$ and this completes the proof.

We shall need the following result in $\$ 7$.

Corollary 3.5. Let $A, J$ and $q_{J}$ be as in Lemma 3.4. If $M$ is a closed right ideal in $A$, then $M^{q} \cap J=(M \cap J)^{q J}$.

Proof. By Lemma 3.1, we have

$$
\left(M^{q} \cap J\right)^{q J}=\left(M^{q} \cap J\right)^{q} \cap J=\operatorname{cl}\left(M+J^{q}\right) \cap J .
$$

Hence by the proof of Lemma 3.4, we have $\left(M^{q} \cap J\right)^{q J} \subset M \cap J$ and so $\left.M^{q} \cap J\right)$ $(M \cap J)^{q J}$. Since $M \cap J \subset M$, it follows that $(M \cap J)^{q} \supset M^{q}$. Therefore $(M \cap J)^{q J}$ $\supset M^{q} \cap J$. Hence $M^{q} \cap J=(M \cap J)^{q J}$.

Now we have the following structure theorem.

Theorem 3.6. Let $A$ be a semisimple quasi-complemented algebra in which every maximal modular right ideal is closed and $x$ belongs to $\mathrm{cl}(x A)$ for all $x \in A$. Then $A$ is the direct topological sum of its minimal closed two-sided ideals, each of which is a simple quasi-complemented algebra.

Proof. By Lemma 3.3, the socle of $A$ is dense in $A$. Therefore by [14, p. 31, Lemma 3.11], $A$ is the topological direct sum of its minimal closed twosided ideals. By Lemma 3.4, each minimal closed two-sided ideal of $A$ is a quasi-complemented algebra and this completes the proof.

Remark. Let $A$ be an algebra. The condition that $x \in \operatorname{cl}(x A)$ for all $x \in A$ is automatically satisfied if $A$ has an approximate identity or $A$ is a semisimple complemented algebra. Also, if $A$ is a semisimple dual algebra, it has this property. 
4. A representation theorem. The following lemma is implicit in [2, p. 40, Proposition 1].

Lemma 4.1. Let $A$ be a semisimple Banach algebra and I a minimal left ideal in $A$. Then

(i) For each closed right ideal $R$ in $A, R I=R \cap I$.

(ii) For each closed subspace $E$ in $I, E=\operatorname{cl}(E A) \cap I$.

Proof. (i). We can write $I=A e$, where $e$ is a minimal idempotent of $A$ (see [14, p. 37]). Let $R$ be a closed right ideal in $A$ and let $x \in R \cap I$. Since $x=$ xe $\in R I$, we have $R \cap I \subset R I$. But $R I \subset R \cap I$ and so $R I=R \cap I$. This proves (i).

(ii) Let $E$ be a closed subspace in $I$ and let $R=\operatorname{cl}(E A)$. Since $E e=E$, we have $E \subset R \cap I$. It follows from (i) that

$$
R \cap I=\operatorname{cl}(E A) I \subset E(e A e)=E e=E .
$$

Therefore $E=\operatorname{cl}(E A) \cap I$ and this completes the proof.

Let $A$ be a primitive quasi-complemented Banach algebra and $I$ a minimal left ideal of $A$. Then $I=A e$ for some minimal idempotent $e$ of $A$. By [10, p. 68, Corollary (2.4.16)], the left regular representation $a \rightarrow T_{a}$ of $A$ is a faithful, continuous, strictly dense representation on $I$. Let $A^{\prime}=\left\{T_{a}: a \in A\right\}$. Then by $[10$, p. 67, Theorem (2.4.12)], the image of the socle of $A$ is the set of all operators of finite rank in $A^{\prime}$. Since by Lemma 3.3 , the socle of $A$ is dense in $A$, it follows that $A$ is a simple algebra (see $[10$, p. 65]).

Lemma 4.2. Let $A$ be a primitive Banach algebra with a quasi-complementor $q$ sucb that $x \in \mathrm{cl}(x A)$ for all $x \in A$. For each closed subspace $E$ in I, let $E^{\prime}=$ $[\mathrm{cl}(E A)]^{q} \cap I$. Then an inner product $(x, y)$ can be introduced in $I$ baving the following properties:

(i) I becomes a Hilbert space under $(x, y)$.

(ii) The norm $|x|=(x, x)^{1 / 2}$ is equivalent to the given norm $\|x\|$ in I.

(iii) If $A$ is infinite-dimensional, then $E^{\prime}$ is the orthogonal complement of $E$ in $I$.

Proof. Let $R=[\mathrm{cl}(E A)]^{q}$. Since $A$ is a simple algebra and since $I A$ is a two-sided ideal in $A, I A$ is dense in $A$. Let $x \in R$. Then

$$
x A=x \operatorname{cl}(I A) \subset \operatorname{cl}(x I A) \subset \operatorname{cl}(R I A) .
$$

Since $x \in \operatorname{cl}(x A)$, it follows that $x \in \operatorname{cl}(R I A)$ and so $R \subset \operatorname{cl}(R I A)$. Clearly $R \supset$ $\mathrm{cl}(R I A)$. Hence $R=\operatorname{cl}(R I A)$. Therefore by Lemma 4.1(i), $R=\operatorname{cl}((R \cap I) A)=$ $\operatorname{cl}\left(E^{\prime} A\right)$. Hence it follows from Lemma 4.1(ii) that

$$
E^{\prime \prime}=\left[\mathrm{cl}\left(E^{\prime} A\right)\right]^{q} \cap I=R^{q} \cap I=\mathrm{cl}(E A) \cap I=E .
$$

If $x \in E \cap E^{\prime}$, then by Lemma 4.1(ii) $x \in \operatorname{cl}(E A) \cap[\operatorname{cl}(E A)]^{q}$ and so $x=0$. 
Therefore $E \cap E^{\prime}=(0)$. If $E_{1}$ and $E_{2}$ are closed subspaces of $I$ such that $E_{1} \subset E_{2}$, then clearly $E_{1}^{\prime} \supset E_{2}^{\prime}$. Therefore by [8, p. 731, Theorem 2], an inner product $(x, y)$ can be introduced in $I$ having properties (i) and (ii). If $A$ is infinite dimensional, then so is $I$. Hence (iii) follows from [8, p. 729, Theorem 1].

We have the following representation theorem.

Theorem 4.3. Let $A$ be a primitive quasi-complemented Banach algebra in which every maximal closed right ideal is modular and $x \in \mathrm{cl}(x A)$ for all $x \in A$. Then there exists a continuous isomorphism of $A$ onto an algebra $A^{\prime}$ of completely continuous operators on a Hilbert space. Also $A$ is a dual algebra.

Proof. Let $I$ be a minimal left ideal in $A$. By Lemma 4.2, $I$ is a Hilbert space. Let $a \rightarrow T_{a}$ be the left regular representation of $A$ on $I$ and $A^{\prime}=$ $\left\{T_{a}: a \in A\right\}$. Then $a \rightarrow T_{a}$ is a continuous isomorphism of $A$ onto $A^{\prime}$. Letting $A^{\prime}$ have the given norm of $A$, we can identify $A$ with $A^{\prime}$. Let $q$ be a given quasicomplementor on $A$ and $R$ a proper closed right ideal of $A$. Since the socle of $A$ is dense in $A$, by [14, p. 37, Lemma 3.1], $R^{q}$ contains a minimal right ideal $M$. It is easy to see that $M^{q}$ is a maximal closed right ideal and so modular by the assumption. Therefore by $\left[14\right.$, p. 38, Lemma 3.3], $l\left(M^{q}\right) \neq(0)$. Since $R \subset M^{q}$, it follows that $l(R) \neq(0)$. Therefore by the proof of $[10$, p. 101, Lemma (2.8.20)], $A$ contains all operators of finite rank on $I$. Hence $A$ is an algebra of completely continuous operators on $I$ (see the proof of $[11$, p. 657, Theorem 7]). By [10, p. 104, Theorem (2.8.23)] $A$ is an annihilator algebra. Since $I$ is reflexive and since $x \in \mathrm{cl}(x A)$, it follows from the proof of $[10, \mathrm{p} .105$, Theorem (2.8.27)] that $A$ is a dual algebra.

Corollary 4.4. Let $A$ be a semisimple quasi-complemented Banach algebra in which $x \in \operatorname{cl}(x A)$ for all $x \in A$. Then $A$ is an annibilator algebra if and only if every maximal closed right ideal of $A$ is modular.

Proof. Suppose every maximal closed right ideal of $A$ is modular. Let $I$ be a minimal closed two-sided ideal of $A$ and $M$ a maximal closed right ideal of $I$. By the proof of Lemma $3.4, M^{q I}$ is a minimal right ideal of $I$ and $A$. Therefore $N=\left(M^{q l}\right)^{q}$ is a maximal modular right ideal of $A$. Since $M^{q I} \oplus N=A$, by [3, p. 462, Lemma 3.1] $N=(1-e) A$ and $M^{q I}=e A$, where $e$ is a minimal idempotent. Since $e \in I, M=\left(M^{q}\right)^{q_{I}}=N \cap I=(1-e) I$. Therefore $M$ is modular. By the proof of Lemma 3.4, we have $x \in \mathrm{cl}(x I)$ for all $x \in I$. Hence by Theorem 4.3, $I$ is an annihilator algebra and so is $A$ by $[10, \mathrm{p} .106$, Theorem (2.8.29)]. The converse of the corollary follows from [10, p. 98, Corollary (2.8.7)].

Theorem 4.5 (we use the notation in Theorem 4.3.). If $A^{\prime}$ is a two-sided ideal of $B(I)$, the set of all continuous linear operators on $I$, then every quasicomplementor $q$ on $A$ is a complementor. 
Proof. By Corollary 3.2, we can assume that $A$ is infinite dimensional. In this proof, we identify $A$ with $A^{\prime}$. Let $R$ be a closed right ideal in $A$. To complete the proof, it suffices to show that $R+R^{q}$ is closed by Lemma 3.1. Let $E=$ $R \cap I$ and let $E^{\prime}=[\mathrm{cl}(E A)]^{q} \cap I$. By Lemma 4.2(iii), $E^{\prime}$ is the orthogonal complement of $E$ in $I$. Denote the orthogonal projection on $E$ by $P$. Let $a \in \mathrm{cl}\left(R+R^{q}\right)$ and write $a=\lim _{n}\left(b_{n}+c_{n}\right)$ with $b_{n} \in R$ and $c_{n} \in R^{q}$. Since $b_{n} I \subset R I=R \cap I=E$, we have $\left(P b_{n}\right)(b)=b_{n}(b)$ for all $b \in I$. Hence $P b_{n}=b_{n}$. Since

$$
c_{n} I \subset R^{q} \cap I=[\mathrm{cl}(R I A)]^{q} \cap I=\operatorname{cl}(E A)^{q} \cap I=E^{\prime},
$$

we have $P c_{n}=0$. By the proof of $\left[2\right.$, p. 41, Theorem 3], we have $\left\|P a-b_{n}\right\| \leq$ $k\left\|a-b_{n}-c_{n}\right\|$, where $k$ is a constant. Hence we have $P a \in R$ and so $a-P a \in R^{q}$. Therefore $a=P a+(a-P a) \in R+R^{q}$. Hence $R+R^{q}$ is closed and this completes the proof.

5. Induced quasi-complementors. In this section, unless otherwise stated, $A$ will be a semisimple Banach algebra with norm $\|\cdot\|$ which is a dense subalgebra of a semisimple Banach algebra $B$ with norm $|\cdot|$. Further $A$ and $B$ have the following properties:

(5.1) There exists a constant $k$ such that $k\|x\| \geq|x|$ for all $x \in A$, i.e., $\|\cdot\|$ majorizes $|\cdot|$.

(5.2) Every proper closed left (right) ideal in $B$ is the intersection of maximal modular (right) ideals in $B$.

Notation. For any subset $E$ of $A, \mathrm{cl}_{A}(E)$ (resp. $\mathrm{cl}(E)$ ) will denote the closure of $E$ in $A$ (resp. B) and $l_{A}(E)$ and $r_{A}(E)$ (resp. $l(E)$ and $r(E)$ ) the left and right annihilators of $E$ in $A$ (resp. $B$ ).

Lemma 5.1. Let $A$ be an annibilator algebra. Then

(i) For each closed right ideal $R$ of $A$, we have $\mathrm{cl}(R) \cap A=r_{A}\left(l_{A}(R)\right)$.

(ii) If $M$ is a closed right ideal of $B$, then $M=\operatorname{cl}(M \cap A)$.

Proof. First we note that $B$ is a dual algebra $[13$, p. 81$]$ and $A$ and $B$ have the same socle $S$ (Lemma 4.1 in [7]).

(i) Let $\left\{e_{a}\right\}$ be the family of all minimal idempotents of $B$ contained in $l(R)$. Since $B$ is a dual algebra, it follows from Lemma 2.1 that $\operatorname{cl}\left(\Sigma_{\alpha} B e_{\alpha}\right)=l(R)$. Since $e_{\alpha} \in l(R) \cap S \subset l(R) \cap A=l_{A}(R)$, we have $\operatorname{cl}\left(l_{A}(R)\right) \supset l(R)$. Clearly $l(R) \supset$ $\mathrm{cl}\left(l_{A}(R)\right)$ and therefore $\operatorname{cl}\left(l_{A}(R)\right)=l(R)=l(\mathrm{cl}(R))$. Hence by the duality of $B$, we have

$$
r_{A}\left(l_{A}(R)\right)=r\left(l_{A}(R)\right) \cap A=r\left(\operatorname{cl}\left(l_{A}(R)\right)\right) \cap A=r(l(\mathrm{cl}(R))) \cap A=\operatorname{cl}(R) \cap A .
$$

This proves (i).

(ii) Let $\left\{e_{\beta}\right\}$ be the family of all minimal idempotents of $B$ contained in $M$. 
By Lemma 2.1, $M=\mathrm{cl}\left(\Sigma_{\beta} e_{\beta} B\right)$. Since each $e_{\beta} B \subset M \cap S \subset M \cap A$, we have $\Sigma_{\beta} e_{\beta} B \subset M \cap A$. It is now easy to see that $M=\operatorname{cl}(M \cap A)$. This completes the proof.

Lemma 5.2. Let $A$ be an annibilator algebra. Then the following statements are equivalent:

(i) A is a dual algebra.

(ii) For each element $x \in A$, we have $x \in \mathrm{cl}_{A}(x A) \cap \mathrm{cl}_{A}(A x)$.

(iii) For each closed right (left) ideal $R$ of $A$, we have $R=\operatorname{cl}(R) \cap A$.

Proof. (i) $\Rightarrow$ (ii). This follows immediately from [10, p. 97, Corollary (2.8.2)].

(ii) $\Rightarrow$ (iii). Suppose (ii) holds. Let $S$ be the socle of $A$. By Lemma 4.1 in [7], $S$ is also the socle of $B$. Let $R$ be a closed right ideal of $A$. We show that $\operatorname{cl}(R) S \subset R$. In fact, let $x \in \operatorname{cl}(R), y \in A$ and $e$ a minimal idempotent in $A$. Let $\left\{x_{n}\right\}$ be a sequence in $R$ such that $x_{n} \rightarrow x$ in $|\cdot|$. By the proof of [13, p. 82, Lemma 3.2], the norms $\|\cdot\|$ and $|\cdot|$ are equivalent on $A e=B e$. Hence it follows easily that $x_{n} y e \rightarrow x y e$ in $\|\cdot\|$. Therefore $x y e \in R$ and so $\operatorname{cl}(R) S \subset R$. Let $a \in$ $\operatorname{cl}(R) \cap A$. Then we have

$$
a \in \mathrm{cl}_{A}(a A)=\mathrm{cl}_{A}(a S) \subset \mathrm{cl}_{A}(\mathrm{cl}(R) S) \subset R .
$$

Hence $\mathrm{cl}(R) \cap A \subset R$. Clearly $R \subset \mathrm{cl}(R) \cap A$ and so $R=\mathrm{cl}(R) \cap A$. This proves (iii).

(iii) $\Rightarrow$ (i). Suppose (iii) holds. Let $R$ be a closed right ideal of $A$. By Lemma 5.1, we have $R=\mathrm{cl}(R) \cap A=r_{A}\left(l_{A}(R)\right)$. Similarly we can show that $J=$ $l_{A}\left(r_{A}(J)\right)$ for all closed left ideals $J$ of $A$. Therefore $A$ is a dual algebra and the proof is complete.

Theorem 5.3. Let $A$ be a dual algebra. Then for every quasi-complementor $p$ on $B$, the mapping $q: R \rightarrow[\mathrm{cl}(R)]^{p} \cap A$ on the closed right ideals $R$ of $A$ is a quasi-complementor on $A$.

Proof. Let $R$ be a closed right ideal of $A$. Since $A$ is a dual algebra, by Lemma 5.2, $R=\operatorname{cl}(R) \cap A$. Therefore

$$
R \cap R^{q}=\mathrm{cl}(R) \cap[\mathrm{cl}(R)]^{p} \cap A=(0) .
$$

By Lemma 5.1, we have $[\mathrm{cl}(R)]^{p}=\mathrm{cl}\left([\mathrm{cl}(R)]^{p} \cap A\right)$. Therefore it follows that

$$
\left(R^{q}\right)^{q}=\left[\mathrm{cl}\left([\mathrm{cl}(R)]^{p} \cap A\right)\right]^{p} \cap A=[\mathrm{cl}(R)]^{p p} \cap A=\operatorname{cl}(R) \cap A=R .
$$

If $R_{1}$ and $R_{2}$ are closed right ideals of $A$ such that $R_{1} \supset R_{2}$, then clearly $R_{1}^{q} C$ $R_{2}^{q}$. Therefore $q$ is a quasi-complementor on $A$.

We now establish the converse of Theorem 5.3.

Theorem 5.4. Let $A$ be a dual algebra. Then for every quasi-complementor $q$ 
on $A$, the mapping $p: M \rightarrow \operatorname{cl}\left([M \cap A]^{q}\right)$ on the closed right ideals $M$ of $B$ is a quasi-complementor on $B$.

Proof. Let $M$ be a closed right ideal of $B$. Then it follows from Lemma 5.2 that $M \cap M^{p} \cap A=[M \cap A] \cap[M \cap A]^{q}=(0)$. Hence it follows from Lemma 5.1 that $M \cap M^{p}=\mathrm{cl}\left(M \cap M^{p} \cap A\right)=(0)$. We also have

$$
\left(M^{p}\right)^{p}=\operatorname{cl}\left(\left[\operatorname{cl}\left([M \cap A]^{q}\right) \cap A\right]^{q}\right)=\operatorname{cl}\left([M \cap A]^{q q}\right)=M .
$$

If $M_{1}$ and $M_{2}$ are closed right ideals of $B$ such that $M_{1} \supset M_{2}$, then clearly $M_{1}^{p} \subset M_{2}^{p}$. Therefore $p$ is a quasi-complementor on $B$ and this completes the proof.

6. Continuous quasi-complementors on $A^{*}$-algebras.

Theorem 6.1. Let $A$ be a dual $A^{*}$-algebra. Then $A$ is a quasi-complemented algebra under the quasi-complementor $q: R \rightarrow l(R)^{*}$.

Proof. Let $R$ be a closed right ideal of $A$. Since $l(R)^{*}=r\left(R^{*}\right)$, by the duality of $A$, we have $\left(R^{q}\right)^{q}=R$. It is easy to see that $q$ has properties (2.1) and (2.3). Therefore $q$ is a quasi-complementor on $A$ and this completes the proof.

It is known that a $B^{*}$-algebra is complemented if and only if it is dual (see [3, p. 463, Theorem 3.6]). A similar result is true for quasi-complemented algebras. In fact we have the following:

Corollary 6.2. Let $A$ be an $A^{*}$-algebra which is a dense two-sided ideal of a $B^{*}$-algebra $B$. Then $A$ is a dual algebra if and only if $A$ is quasi-complemented and $x \in \mathrm{cl}(x A)$ for all $x \in A$.

Proof. Suppose $A$ is quasi-complemented and $x \in \operatorname{cl}(x A)$ for all $x \in A$. Let $e$ be a minimal idempotent of $A$. Clearly $A e=B e$. Therefore by Lemma 3.3 and Theorem 4.3 in [7], $A$ is an annihilator algebra. Hence by Lemma 5.2, $A$ is a dual algebra. The converse of the corollary follows from Theorem 6.1 and Lemma 5.2.

Corollary 6.3. Let $A$ be a $B^{*}$-algebra. Then $A$ is a dual if and only if $A$ is quasi-complemented.

Proof. Since a $B^{*}$-algebra has an approximate identity, it follows that $x \in$ $\operatorname{cl}(x A)$. Therefore Corollary 6.3 follows immediately from Corollary 6.2.

Lemma 6.4. Let $A$ be an annibilator semisimple Banach algebra with a quasi-complementor $q$. Then for every maximal closed right ideal $R$ of $A$, there exists a unique minimal idempotent $f$ such that $R^{q}=f A$ and $R=(1-f) A$.

Proof. By [10, p. 97, Theorem (2.8.5)], $R$ is a maximal modular right ideal of $A$. Since $R+R^{q}=A$, by [3, p. 462, Lemma 3.1] we have the desired result. 
Definition. Let $A$ be a quasi-complemented Banach algebra. A minimal idempotent $f$ in $A$ is called a $q$-projection if $(f A)^{q}=(1-f) A$.

We now introduce the concept of continuous quasi-complementor on annihilator $A^{*}$-algebras. This is similar to the concept of cont inuous complementor on $B^{*}$-algebras (see [3, p. 463, Definition 3.7]).

Definition. Let $A$ be an annihilator $A^{*}$-algebra with a quasi-complementor $q$. Let $E$ denote the set of all hermitian minimal idempotents and $E_{q}$ the set of all $q$-projections in $A$. For each $e \in E$, let $Q(e)$ be the unique element of $E_{q}$ such that $Q(e) A=e A$ (Lemma 6.4). The mapping $Q: e \rightarrow Q(e)$ is called the $q$-derived mapping of $E$ into $E_{q}$. The quasi-complementor $q$ is said to be continuous if $Q$ is continuous in the relative topologies of $E$ and $E_{q}$ induced by the given norm on $A$.

Remark 1. Since by $[10$, p. 261, Lemma (4.10.1)] every minimal right ideal of $A$ is of the form $e A$ with a unique $e \in E$, it follows that $Q$ maps $E$ onto $E_{q}$.

Remark 2. Let $A$ and $q$ be as in Theorem 6.1. Then $E=E_{q}$ and so the $q$-derived mapping $Q$ of $q$ is the identity mapping. Hence $q$ is uniformly continuous.

For commutative dual $A^{*}$-algebras, the study of quasi-complementor becomes very trivial.

Theorem 6.5. Let $A$ be a commutative dual $A^{*}$-algebra. Then there is only one quasi-complementor $q$ on $A ; q$ is uniformly continuous.

Proof. Let $B$ be the completion of $A$ in an auxiliary norm. We use the notation introduced in $\$ 5$. The existence of a quasi-complementor on $A$ is given by Theorem 6.1. Let $q$ be any given quasi-complementor on $A$. By Theorem 5.6, $q$ induces a quasi-complementor $p$ on $B$. Let $M$ be a closed ideal in $B$. Since $M \cap M^{q}=(0)$, it follows from [10, p. 259, Corollary (4.9.22)] that $M+M^{q}$ is a closed ideal in $B$. Therefore, by Lemma 3.1, $M+M^{p}=B$. Since $M M^{p} \subset M \cap M^{p}=$ (0), $M^{p} \subset l(M)=r(M)$. Since $M+l(M)=B$, it follows that $M^{p}=l(M)$. Let $R$ be an ideal in $A$. Then we see that $R=R^{*}$ and $R^{q}=[\mathrm{cl}(R)]^{p} \cap A=l_{A}(R)$. Therefore $q$ is uniquely determined. By Remark 2, $q$ is uniformly continuous and this completes the proof.

Corollary 6.6. Let $A$ be a commutative dual $A^{*}$-algebra which is a dense two-sided ideal of a $B^{*}$-algebra. Then there is a unique complementor $q$ on $A$; $q$ is uniformly continuous.

Proof. This follows easily from Theorem 6.5, [4, p. 233, Theorem 3.8] and [9, p. 30, Theorem 16].

7. Quasi-complementors on $B^{*}$-algebras. In this section, unless otherwise stated, $A$ will be a $B^{*}$-algebra with a quasi-complementor $q$. By Corollary 6.3, $A$ is a dual a lgebra. 
Let $H$ be a Hilbert space with inner product (, ). If $x$ and $y$ are elements of $H$, then $x \otimes y$ will denote the operator on $H$ given by the relation $(x \otimes y)(b)=$ $(b, y) x$ for all $b \in H . L C(H)$ will denote the algebra of all completely continuous linear operators on $H$. If $A$ is a simple dual $B^{*}$-algebra, then it is well known that $A=L C(H)$ for some Hilbert space $H$. $H$ can be chosen as a minimal left ideal in $A$ with the inner product given in [10, p. 261, Theorem (4.10.3)].

Lemma 7.1. Let $A$ be a simple $B^{*}$-algebra. Then every quasi-complementor $q$ on $A$ is a complementor.

Proof. Since $A$ has the form $L C(H)$, it follows from Theorem 4.5 that $q$ is a complementor on $A$.

Notation. Let $A=L C(H)$. For every closed subspace $X$ of $H$, let $J(X)=$ $\{a \in A: a(H) \subset X\}$. For every closed right ideal $R$ of $A$, let $S(R)$ be the smallest closed subspace of $H$ that contains the range $a(H)$ of each operator $a$ in $R$.

Let $A=L C(H)$. For each closed right ideal $R$ of $A$, by Lemma 7.1, the projection $P_{R}$ on $R$ along $R^{q}$ is continuous. Let $P_{R}^{\prime}$ be the projection on $S(R)$ along $S\left(R^{q}\right)$. Since by [3, p. 464, Lemma 4.1], $S(R) \oplus S\left(R^{q}\right)=H$, it follows that $P_{R}^{\prime}$ is continuous.

Lemma 7.2. Let $R$ be a closed right ideal of $A=L C(H)$. Then $\left\|P_{R}\right\|=\left\|P_{R}^{\prime}\right\|$.

Proof. Let $k>0$ be given. Choose $x \in A$ such that $\|x\| \leq 1$ and $\left\|P_{R}(x)\right\| \geq$ $\left\|P_{R}\right\|-k / 2$. Hence there exists some $b \in H$ such that $\|b\| \leq 1$ and $\left\|\left(P_{R}(x)\right)(b)\right\|>$ $\left\|P_{R}\right\|-k$. Write $x=y+z$ with $y \in R$ and $z \in R^{q}$. Then $y(b) \in S(R)$ and $z(b) \epsilon$ $S\left(R^{q}\right)$ and so

$$
\left\|P_{R}^{\prime}(x(b))\right\|=\|y(b)\|=\left\|\left(P_{R}(x)\right)(b)\right\|>\left\|P_{R}\right\|-k .
$$

Since $\|x(b)\| \leq 1$ and $k$ is arbitrary, it follows that $\left\|P_{R}^{\prime}\right\| \geq\left\|P_{R}\right\|$. By using [3, p. 464 , Lemma 4.1] and a similar argument, we can show that $\left\|P_{R}^{\prime}\right\| \geq\left\|P_{R}\right\|$. Therefore $\left\|P_{R}\right\|=\left\|P_{R}^{\prime}\right\|$.

Lemma 7.3. Suppose $A=L C(H)$ with $\operatorname{dim} H \geq 3, q$ a continuous quasicomplementor on $A$ and $R$ a closed right ideal of $A$. If $\left\|P_{R}\right\|>k$ for some constant $k$, then there exists a q-projection $f \in R$ sucb that $\|f\|>k$.

Proof. By Lemma 7.2, $\left\|P_{R}^{\prime}\right\|>k$. Hence there exists an element $b \in H$ such that $\|b\|=1$ and $\left\|P_{R}^{\prime}(b)\right\|>k$. Write $b=u+v$ with $u \in S(R)$ and $v \in S\left(R^{q}\right)$. It is clear that $u \neq 0$. Let $Q$ be a $q$-representing operator on $H$ (see [3, p. 467, Definition 5.4]) and put $f=(u \otimes Q u) /(u, Q u)$. Then $f$ is a $q$-projection (see [3, p. 467]). Since $u \in S(R), f \in R$. Let $\langle x, y\rangle=(x, Q y)$ for all $x, y \in H$. Since $q$ is a continuous complementor, by the proof of [3, p. 473, Theorem 6.11], $S(R)$ is the orthogonal complement of $S\left(R^{q}\right)$ in $H$ relative to the inner product $\langle x, y\rangle$. Since 
$v \in S\left(R^{q}\right)$ and since $u \in S(R)$, we have $(v, Q u)=\langle v, u\rangle=0$ and therefore $f(b)=u$. Hence we have $\|f(b)\|=\|u\|=\left\|P_{R}^{\prime}(b)\right\|>k$. Since $\|b\|=1,\|f\|>k$ and this completes the proof.

Let $A$ be a $B^{*}$-algebra with a quasi-complementor $q$. Let $\left\{I_{\lambda}: \lambda \in \Lambda\right\}$ be the family of all minimal closed two-sided ideals of $A$. Since $A$ is a dual $B^{*}$-algebra, $A=\left(\Sigma_{\lambda} I_{\lambda}\right)_{0}$, the $B^{*}(\infty)$-sum of $\left\{I_{\lambda}: \lambda \in \Lambda\right\}$. Since each $I_{\lambda}$ is a simple dual $B^{*}$ algebra, $I_{\lambda}=L C\left(H_{\lambda}\right)$ for some Hilbert space $H_{\lambda}(\lambda \in \Lambda)$. By Corollary 3.5, $q$ induces a quasi-complementor $q_{\lambda}$ on each $I_{\lambda}$. By Lemma 7.1, $q_{\lambda}$ is a complementor on $I_{\lambda}$.

Let $E$ (resp. $E_{\lambda}$ ) be the set of all hermitian minimal idempotents in $A$ (resp. $I_{\lambda}$ ) and let $E_{q}$ (resp. $E_{q}^{\lambda}$ ) be the set of all $q$-projections in $A$ (resp. $I_{\lambda}$ ). Clearly $E_{\lambda}=E \cap I_{\lambda}$ and $E_{q}^{\lambda}=E_{q} \cap I_{\lambda}(\lambda \in \Lambda)$.

Lemma 7.4. A quasi-complementor $q$ on $A$ is continuous if and only if each $q_{\lambda}$ is continuous.

Proof. By a similar argument in [3, p. 464, Theorem 3.9], we have the desired result.

Lemma 7.5. Let $A$ be a $B^{*}$-algebra which has no minimal left ideal of dimension less than three and $q$ a quasi-complementor on $A$. If $E_{q}$ is a closed and bounded subset of $A$, then $q$ is a complementor on $A$.

Proof. For each closed right ideal $R_{\lambda}$ of $L C\left(H_{\lambda}\right)$, let $P_{R_{\lambda}}$ be the projection on $R_{\lambda}$ along $R_{\lambda}^{q}$. Let

$$
k_{\lambda}=\sup \left\{\left\|P_{R_{\lambda}}\right\|: R_{\lambda} \subset L C\left(H_{\lambda}\right)\right\} \quad(\lambda \in \Lambda)
$$

and let

$$
k=\sup \left\{k_{\lambda}: \lambda \in \Lambda\right\}
$$

We show that $k$ is finite. Suppose this is not so. Then for each positive integer $n$, there exists some $k_{n} \in\left\{k_{\lambda}: \lambda \in \Lambda\right\}$ such that $k_{n}>n$. Hence there exists a closed right ideal $R_{n} \subset L C\left({ }_{n}\right)$ such that $\left\|P_{R_{n}}\right\|>n$. Since $E_{q}^{n}=E_{q} \cap I_{n}$, it follows immediately from the assumption that $E_{q}^{n}$ is a closed and bounded subset of $I_{n}$. Since $q_{n}$ is a complementor on $I_{n}$, by $\left[12\right.$, p. 257, Theorem 3], $q_{n}$ is continuous. Since $\left\|P_{R_{n}}\right\|>n$, it follows from Lemma 7.3 that there exists some $f_{n} \epsilon$ $E_{q}^{n} \subset E_{q}$ such that $\left\|f_{n}\right\|>n(n=1,2, \ldots)$. This contradicts the boundedness of $E_{q}$ and shows that $k$ is finite.

Let $M$ be a closed right ideal of $A$ and let $M_{\lambda}=M \cap I_{\lambda}(\lambda \in \Lambda)$. Since $A=$ $\left(\Sigma_{\lambda} I_{\lambda}\right)$, we see that $M=\left(\Sigma_{\lambda} M_{\lambda}\right)_{0}$. Since by Corollary $3.5, M^{q} \cap I_{\lambda}=M_{\lambda}^{q} \lambda$, we have

$$
M^{q}=\left(\Sigma_{\lambda} M^{q} \cap I_{\lambda}\right)_{0}=\left(\Sigma_{\lambda} M_{\lambda}^{q}{ }_{\lambda}\right)_{0} .
$$


Let $x=\left(x_{\lambda}\right) \in A$ and write $x_{\lambda}=y_{\lambda}+z_{\lambda}$, where $y_{\lambda} \in M_{\lambda}$ and $z_{\lambda} \in M_{\lambda}^{q}{ }_{\lambda}$. Then $\left\|y_{\lambda}\right\|=\left\|P_{M_{\lambda}}{ }_{\lambda}\right\| \leq k\left\|x_{\lambda}\right\|(\lambda \in \Lambda)$. Since $k$ is finite, it follows that $\left(y_{\lambda}\right) \epsilon$ $\left(\Sigma_{\lambda} M_{\lambda}\right)_{0}=M$. Similarly we have $\left(z_{\lambda}\right) \in M^{q}$. Therefore $A=M+M^{q}$ and so $q$ is a complementor on $A$.

We can now prove the main result of this section.

Theorem 7.6. Let $A$ be a $B^{*}$-algebra which has no minimal left ideal of dimension less than three and $q$ a quasi-complementor on A. If $q$ is uniformly continuous, then it is a complementor.

Proof. By Lemma 7.5, it suffices to show that $E_{q}^{\lambda}$ is a closed and bounded subset of A. By Lemma 7.4 and [12, p. 257, Theorem 3] each $E_{q}^{\lambda}$ is closed and bounded. Hence it follows that $E_{q}$ is closed. It remains to show that $E_{q}$ is bounded. Suppose this is not so. Then we can choose a sequence of $q$-projections $f_{n}$ such that $f_{n} \in E_{q}^{n}$ and $\left\|f_{n}\right\|>n(n=1,2, \cdots)$. Let $T_{n}$ be a $q$-representing operator on $H_{n}$. Then by [3, p. 470, Theorem 6.4], $T_{n}$ is a continuous positive linear operator with inverse $T_{n}^{-1}$. We may assume that $\left\|T_{n}^{-1}\right\|=1$ for all $n$ (see $[3$, p. 472, Corollary 6.10]). We can write

$$
f_{n}=\left(u_{n} \otimes T_{n} u_{n}\right) /\left(u_{n}, T_{n} u_{n}\right)
$$

where $u_{n} \in H_{n}$ and $\left\|u_{n}\right\|=1(n=1,2, \cdots)$ (see [3, p. 467]). Since

$$
\inf \left\{\left(b_{n}, T_{n} b_{n}\right):\left\|b_{n}\right\|=1 \text { and } b_{n} \in H_{n}\right\}=\left\|T_{n}^{-1}\right\|^{-1}=1
$$

if follows from $(*)$ that $\left\|T_{n} u_{n}\right\|>n(n=1,2, \cdots)$. Let $Q$ be the $q$-derived mapping of $q$. By using the argument in [3, p. 477, Theorem 7.4], we can find minimal idempotents $a_{n}, b_{n} \in E$ such that $\left\|a_{n}-b_{n}\right\| \rightarrow 0$ and $\left\|Q\left(a_{n}\right)-Q\left(b_{n}\right)\right\| \rightarrow \infty$. This contradicts the uniform continuity of $Q$. Therefore $E_{q}$ is bounded and this completes the proof.

Remark. Let $B$ and $p$ be given in [1, p. 396, Example 1]. Then $p$ is a continuous quasi-complementor on $B$. But $p$ is not a complementor. Therefore a continuous quasi-complementor may not be uniformly continuous by Theorem 7.6. However a continuous complementor on a $B^{*}$-algebra is uniformly continuous (see [1] and [3]).

Corollary 7.7. Let $A$ be as in Theorem 7.6. Then a quasi-complementor $q$ on $A$ is uniformly continuous if and only if $E_{q}$ is a closed and bounded subset of $A$.

Proof. The corollary follows immediately from Theorem 7.6 and $[12$, p. 257, Theorem 3]. 


\section{REFERENCES}

1. F. E. Alexander, Representation theorems for complemented algebras, Trans. Amer. Math. Soc. 148 (1970), 385-397. MR 43 \#916.

2. - On complemented and annihilator algebras, Glasgow J. Math. 10 (1969), 38-45. MR $39 \# 6086$.

3. F. E. Alexander and B. J. Tomiuk, Complemented $B^{*}$-algebras, Trans. Amer. Math. Soc. 137 (1969), 459-480. MR 38 \#5009.

4. G. F. Bachelis, Homomorphisms of annihilator Banach algebras, Pacific J. Math. 25 (1968), 229-247. MR $39 \# 6076$.

5. B. A. Barnes, Modular annihilator algebras, Canad J. Math. 18 (1966), 566-578. MR 33 \#2681.

6. J. Dixmier, Les $C^{*}$-algèbres et leurs représentations, Cahiers Scientifiques, fasc. 29, Gauthier-Villars, Paris, 1964. MR 30 \#1404.

7. T. Husain and P. K. Wong, On generalized right modular complemented algebras, Studia Math. 45 (1972) 37-42.

8. S. Kakutani and G. W. Mackey, Ring and lattice characterizations of complex Hilbert space, Bull. Amer. Math. Soc. 52 (1946), 727-733. MR 8, 31.

9. T. Ogasawara and K. Yoshinaga, Weakly completely continuous Banach *algebras, J. Sci. Hirosh ima Univ. Ser. A. 18 (1954), 15-36. MR 16, 1126.

10. C. E. Rickart, General theory of Banach algebras, University Series in Higher Math., Van Nostrand, Princeton, N. J. 1960. MR 22 \#5903.

11. B. J. Tomiuk, Structure theory of complemented Banach algebras, Canad. J. Math. 14 (1962), $651-659$. MR $26 \# 626$.

12. P. K. Wong, Continuous complementors on $B^{*}$-algebras, Pacific J. Math. 33 (1970), 255-260.

13, - - On the Arens product and annihilator algebras, Proc. Amer. Math. Soc. 30 (1971), 79-83.

14. B. Yood, Ideals in topological rings, Canad. J. Math. 16 (1964), 28-45. MR 28 $\# 1505$.

DEPARTMENT OF MATHEMATICS, McMASTER UNIVERSITY, HAMILTON, ONTARIO, CANADA (Current address of T. Husain)

Current address (P. K. Wong): Department of Mathematics, Seton Hall University, South Orange, New Jersey 07079 